\title{
Electromyographic study of the rigidospasticity of athetosis
}

\author{
COLIN J. ANDREWS ${ }^{1}$, PETER NEILSON ${ }^{2}$, AND L. KNOWLES \\ From the Division of Neurology, The Prince Henry Hospital, and the School of Medicine, \\ University of New South Wales, Sydney, Australia
}

SUMMARY The static and dynamic components of the tonic stretch reflex and shortening reactions $\stackrel{\mathbb{D}}{\varrho}$ have been studied in 10 patients with athetosis. EMG activity could be recorded only from the is biceps muscle when the patient was at rest. The dynamic stretch reflex increased with the velocity of $\vec{\circ}$ stretching in all muscles examined except the biceps. The biceps stretch reflex was found to be inhibited by increasing muscle length, whereas the stretch reflexes of triceps, hamstrings, and quadriceps muscles were facilitated by increasing muscle length. Reinforcement increased resting activity in the biceps and the dynamic shortening reaction of the triceps muscle. Both these effects were suppressed by the action of phenoxybenzamine. Although phenoxybenzamine was shown to $\dot{\omega}_{\sigma}$ reduce muscle tone in a double-blind controlled trial, no corresponding improvement was detected in involuntary movements or the patients' performance in a tracking test. The differences betwee $\overline{0} \dot{\phi}$ the pattern of hypertonus in athetosis, Parkinson's disease, spasticity, and activated normal subject are presented in discussion.

Narabayashi, Nagahata, Nagao, and Shimazu (1965) defined athetosis as 'a clinical manifestation of fluctuating rigidity or rigidospasticity', Herz (1931) considered that athetotic patients were always hypertonic in the intervals between involuntary movements, whereas Hoefer and Putnam (1940) observed no rigidity when the patient was at rest.

Activation (mental activity, emotional disturbance, or reinforcement by voluntary muscular contraction) is known to produce or aggravate involuntary movements of basal gangliar origin. The transmitter substances employed by the ascending reticular activating system which mediates arousal are uncertain, but noradrenaline is known to have actions on reticular neurones of the brain-stem when applied iontophoretically (Bradley and Wolstencraft, 1965) and the turnover of noradrenaline in the brain is increased under immobilization stress (Corrodi, Fuxe, Lidbrink, and Olsen, 1971). The anatomy and function of the descending noradrenergic

\footnotetext{
1 Edwin and Daisy Street Fellow in Neurology.

2 Centre Industries Research Scholar.

${ }^{3}$ Research Assistant, National Health and Medical Research Council of Australia.
}

system are better known. The neurones of the system are localized to the caudal medulfa. (Dahlström and Fuxe, 1965) and the fibre descend in the lateral and anterior funiculi of the spinal cord (Carlsson, Falck, Fuxe, and Hillarp, 1964). Carlsson was unable to find noradrenaline in any neurones of spinal cord origin. Administration of L-dopa causes the release of noradrenaline from descending adrenergic fibres in the acutely spinalized cat (Andén, Jukes, and Lundberg, 1966; Andén, Jukes, Lundberg, and Vyklicky, 1966), which can be abolished by phenoxybenzamine (Andén et al., 1966). The action of the descending noradrenergic pathway on segmental mechanisms is complex. On the efferent side, alpha motoneurone excitability is increased (Baker and 8 Anderson, 1970) and, in flexor muscles, static $₹$ fusimotor activity is increased, whereas dynamic 으 fusimotor activity is decreased (Bergmans and $D$ Grillner, 1968). In extensor muscles, fusimotor drive of both dynamic and static motoneurones $N$ is increased (Grillner, 1969). Alteration of reflex activity by L-dopa in acute spinalized cats can be summarized as a decrease in short-latency 
reflexes and transmission in group $\mathrm{Ib}$ fibres and an increase in long-latency flexor reflexes without significant alteration in group Ia nerve fibre transmission (Table 1).

Ethyl alcohol and barbiturates are only mildly effective in reducing athetosis and the amount of intravenous amylobarbitone necessary to abolish

TABLE 1

ACTION OF DESCENDING NORADRENERGIC PATHWAY ON SEGMENTAL MECHANISMS

\begin{tabular}{lcc}
\hline & Extensor & Flexor \\
\hline Excitability of & & \\
Alpha motoneurones & $\uparrow$ & $\uparrow$ \\
Static fusimotor neurones & $\uparrow$ & $\uparrow$ \\
Dynamic fusimotor neurones & $\uparrow$ & $\downarrow$ \\
Flexor reflexes short latency & $\downarrow$ & $\uparrow$ \\
Group Ib reflex transmission & $\uparrow$ & $\downarrow$ \\
Group Ia reflex transmission & Unchanged & Unchanged \\
\hline
\end{tabular}

choreoathetosis nearly always induces sleep (Bergman, Nathanson, and Bender, 1953). The therapeutic usefulness of sedatives is thus limited by drowsiness.

In view of the importance of activation in aggravating athetosis, and the probable part played by noradrenaline in the mechanism of activation, it was considered worthwhile to test an alpha-adrenergic blocking agent (phenoxybenzamine) in the treatment of this condition. Since there has been little attempt to analyse the disordered physiology of athetosis in the past, it became necessary to study the stretch reflexes and shortening reactions in athetosis and their response to activation as a baseline for assessment of the therapeutic action of phenoxybenzamine.

\section{METHODS}

All patients suffered from an athetosis-spasticity syndrome as the result of birth trauma or hypoxia. Of the 10 patients, seven were male and three were female, aged 22 to 41 years. All patients had mild weakness of pyramidal tract type, with increased muscle tone in the limb examined, while athetoid movements became apparent during activity. All patients were employed in a sheltered workshop but differed in functional capacity and only one was in- capable of walking unassisted. The study was of crossover design and capsules of phenoxybenzamine $10 \mathrm{mg}$ or placebo were administered as a single dose of two capsules each night, increasing to three capsules if no side-effects were encountered. The allocation of the patients to treatment or placebo groups was determined by a system of random numbers and the key was held by the hospital pharmacist.

The principles of electromyographic methods of assessment have been described elsewhere (Burke, Gillies, and Lance, 1970; Burke, Andrews, and Gillies, 1971). Linear stretching movements were used to determine the threshold and sensitivity of the stretch reflex to the velocity of stretch, while sinusoidal stretching at different joint positions was used to determine the response to changes in muscle length. The average sinusoidal movements were used to calculate the phase relationships of the stretch reflexes and shortening reactions during sinusoidal stretching as described below.

The electromyogram (EMG) of biceps, triceps, hamstrings, and quadriceps muscles was recorded by surface electrodes, the position of electrodes being the same for assessments during each of the two periods. Movement artefact was excluded by monitoring each response on an oscilloscope. The quadriceps and hamstrings muscles were stretched by passive movements of the knee joint while the patient lay in the prone position; the biceps and triceps muscles were examined with the patient in the supine position. Joint angle was recorded by a goniometer and monitored on an oscilloscope together with the EMG tracings. The goniometer output was differentiated (time constant $5 \mathrm{msec}$ ) to produce a voltage proportional to angular velocity. The EMG potentials were integrated (time constant $0.2 \mathrm{sec}$ ) to provide a measure of the stretch reflex. The velocity of stretch, joint angle, EMG and integrated EMG (IEMG) were recorded on a four-channel type, Offner dynograph and a Grass polygraph. In recording the reflex response to sinusoidal movement the EMG of biceps and triceps muscles was amplified, full-wave rectified and fed into the multiple input unit of an ND 801 Enhancetron. Averaging by the Enhancetron was triggered from the goniometer output when a preset trigger voltage had been exceeded. The output of the Enhancetron was displayed on a Tektronix 531A oscilloscope and photographed with a Polaroid Land camera. The method is described in more detail by Burke et al. (1971).

The line of best fit of the relationship between velocity of stretch and the height of the integrated EMG was drawn to compare the dynamic component of the tonic stretch reflex during periods of treatment with placebo and phenoxybenzamine. The minimal 
velocity of stretch necessary to produce activity in the EMG was expressed as the threshold velocity of the dynamic stretch reflex and the 'dynamic sensitivity' was defined as the increase in IEMG produced by an increase in velocity of stretch by $100^{\circ} / \mathrm{sec}$, expressed as a percentage of that evoked at $400^{\circ} / \mathrm{sec}$. Since the latter depends upon both static and dynamic factors, the dynamic sensitivity indicates the proportion of the reflex response which may be attributed to velocity-dependent factors. The phase relationship of the reflex response to the position of maximal stretch was calculated by relating the peak of the EMG activity to the most stretched position of the muscle. If the amplitude of the reflex depends solely on muscle length there will be no phase lead. A velocity-dependent reflex will tend to become maximal 90 degrees in advance of the most stretched position. A phase lead greater than 90 degrees is assumed to be due to length-dependent inhibition. The amount of phase lead, if any, will thus provide an indication of the relative contributions of velocity of stretch, and facilitation or inhibition produced by changes in muscle length. Reinforcement was produced by asking the patient to clench the fist on the side opposite to the limb being examined, and the degree of voluntary muscular contraction was monitored by EMG recordings.

A quantitative description of involuntary athetoid movements was obtained from an analysis of visual tracking performed by 10 athetoid patients. The patient lay supine on a couch with the right arm strapped into a frame which constrained movement to flexion-extension about the elbow. A goniometer attached to the frame enabled the elbow joint angle to be recorded on a four-channel Grass polygraph. Disc surface electrodes were placed $5 \mathrm{~cm}$ apart over the belly of the biceps brachii muscle so that EMG and IEMG (time constant $0 \cdot 16 \mathrm{sec}$ ) could be recorded on the polygraph. The signal from the goniometer controlled the vertical position of a horizontal line on an oscilloscope screen which provided the patient with a visual feedback of elbow angle. A second line, not so bright and slightly defocused, was moved up and down on the screen in an arbitrary manner by the experimenter. The task for the patient was to move his arm-that is, change elbow angle-so as to keep the two lines on the oscilloscope screen superimposed. Signals proportional to the position of the target line and position of elbow angle were recorded on the polygraph. This visual pursuit tracking test lasted for about $5 \mathrm{~min}$, during which time the target line was moved with a variable velocity which at times exceeded the patients' tracking ability.

The target and elbow angle signals were analysed by correlation and spectral analysis techniques
(Blackman and Tukey, 1959; Jenkins and Watts, $\underset{\mathbb{Q}}{Z}$ 1968). Cross-correlation between target position and elbow angle separated the involuntary athetoid of movements from those correlated with target move- $\frac{0}{0}$ ments. The power spectrum of the involuntary move- $D$ ments made by the athetoid patients during the visual tracking test was thus computed. The test was repeated after the patient had been treated with placebo and after treatment with phenoxybenzamine.

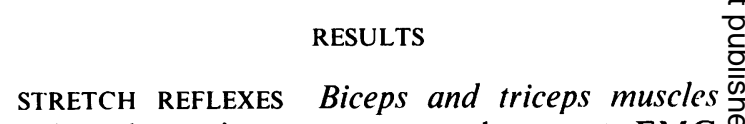
When the patient was apparently at rest, EMG $\stackrel{\mathbb{D}}{\Omega}$ potentials could be recorded from the biceps $\%$ muscle in eight out of 10 patients but from the $\overrightarrow{0}$ triceps muscle in only one patient. During the stretching movement, the dynamic stretch reflex $\vec{\omega}$ increased with the velocity of stretch of the triceps muscle, the relationship clearly being linear in seven out of 10 patients (Fig. 1). No similar relationship was found for the biceps muscle and in some patients the dynamic stretch refle actually diminished as the velocity of stretch w然음 increased (Fig. 2).

In the biceps muscle the static and dynamix stretch reflexes were maximal at the flexed
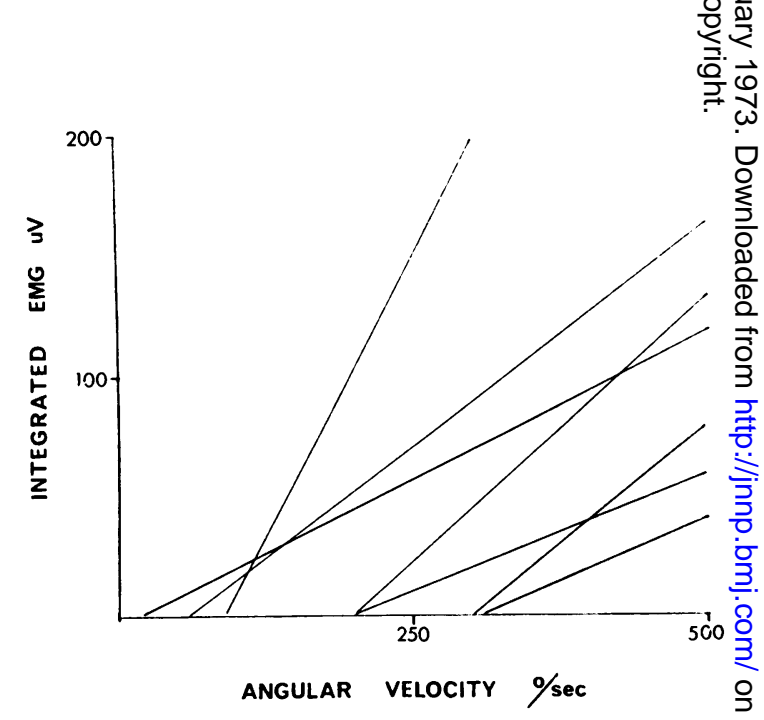

FIG. 1. The relationship of the dynamic stretch reflex of the triceps muscle to velocity of stretch. Note that high threshold velocity is generally associated with aN stretch reflex of low amplitude, whereas a low threshold $\mathrm{N}$ velocity is associated with a stretch reflex of high amplitude. 
position of the elbow in seven out of the eight patients examined (Figs 2 and 3), while the dynamic stretch reflex in the triceps muscle was maximal at the fully stretched position in all patients.

The EMG response to sinusoidal stretching of the biceps muscle, averaged over 20 cycles at

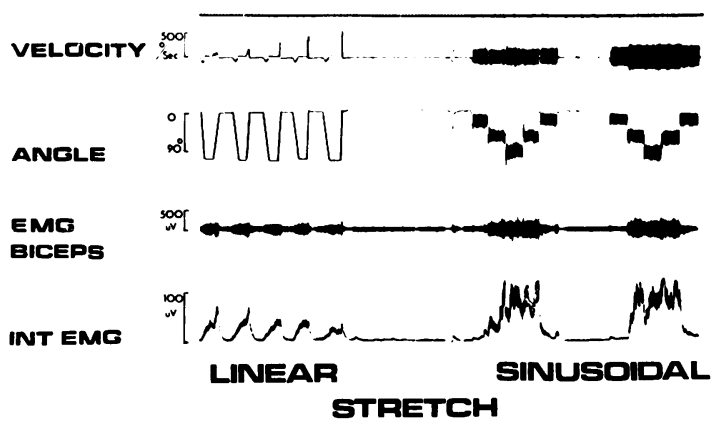

FIG. 2. Static and dynamic EMG responses recorded from the biceps brachii muscle of an athetotic patient. Linear stretching movements on the left side of the Figure evoke a static response in the shortened position $\left(90^{\circ}\right)$ and a dynamic stretch reflex during the stretching movement $\left(90-0^{\circ}\right)$, which fatigues as the movement is repeated with increasing velocity. There is little static reflex activity to maintained stretch. Sinusoidal stretching at different elbow positions (right side of Figure) demonstrates that the dynamic stretch reflex is maximal in the shortened position of the biceps muscle and is suppressed when the muscle is stretched.
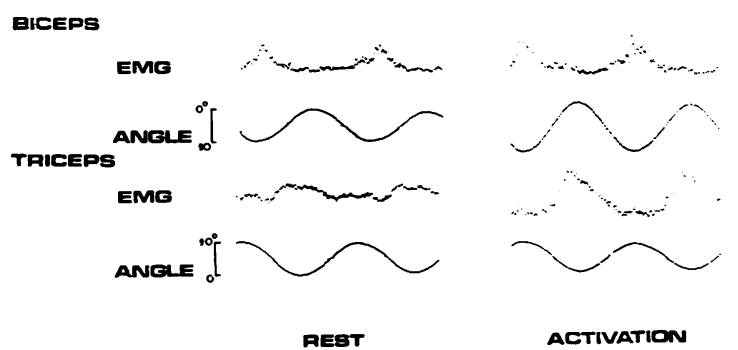

1 CPS
$1 / \mathrm{sec}$ through a range of elbow joint movement of $90^{\circ}$ (from flexion to extension), showed a phase lead of $90^{\circ}$ or greater in the four patients in whom this was examined. In contrast, the EMG of the triceps muscle showed a phase lead of less than $90^{\circ}$ (Fig. 4).

Hamstrings and quadriceps muscles No EMG potentials could be recorded from either muscle

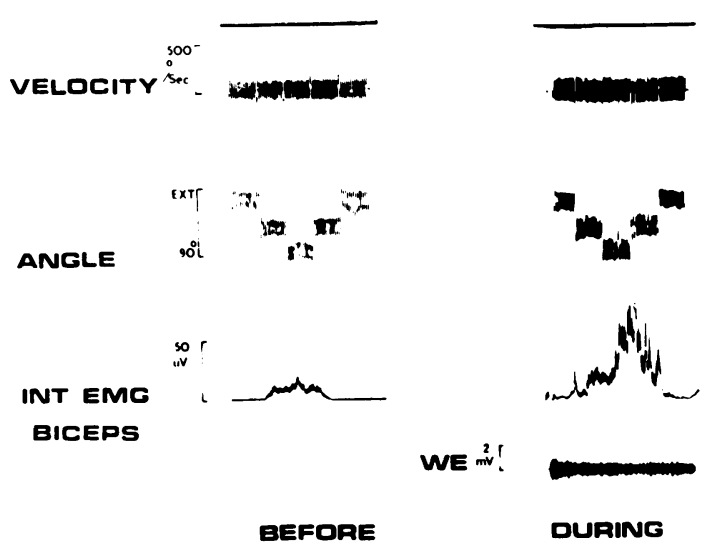

\section{activation}

FIG. 3. Augmentation of the dynamic stretch reflex of the biceps muscle by reinforcement. The reflex remains maximal in the flexed position of the elbow. The EMG of the contralateral wrist extensor (WE) is mounted during activation as a means of monitoring the degree of reinforcement.

FIG. 4. The response of the stretch reflexes and shortening reactions during sinusoidal stretch of the biceps and triceps muscles, showing the effect of activation and ischaemia of the elbow joint and forearm. The phase lead of biceps stretch reflex on the most stretched position is approximately $160^{\circ}$ and is slightly increased during elbow ischaemia activation and ischaemia. In the triceps muscle the peak of the EMG activity occurred during shortening and augmented during activation and ischaemia. 


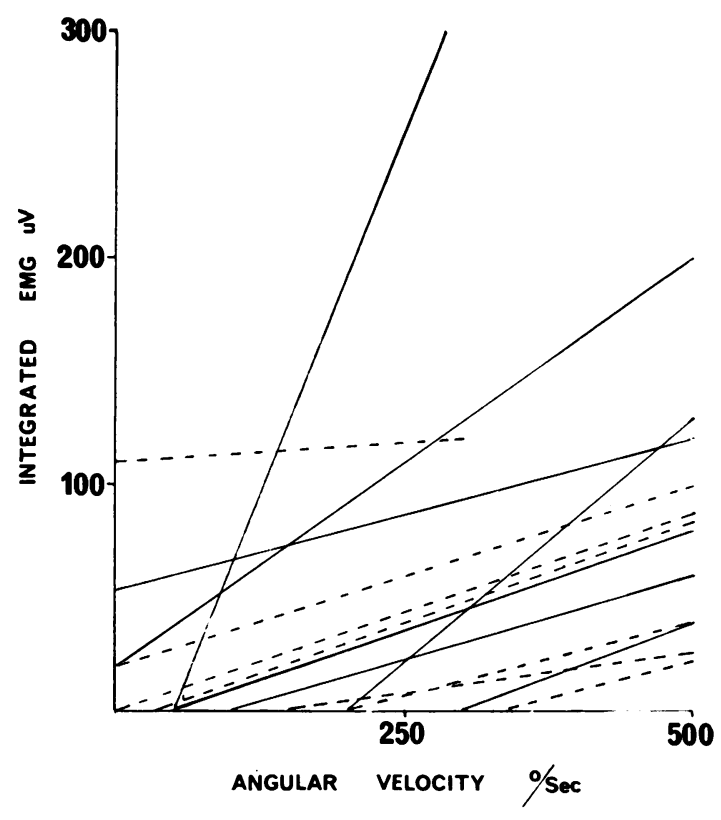

FIG. 5. The relationship of the dynamic shortening reaction of the triceps muscle to increasing velocities of shortening during the placebo (continuous line) and phenoxybenzamine periods (broken line). A moderate reduction of the dynamic shortening reaction occurred in all but one patient. with the patient at rest. A dynamic stretch reflex $\overline{\mathrm{D}}$ was recorded from the hamstrings in all patients $\overline{ }$ and from the quadriceps muscle in nine out of $10, \stackrel{0}{工}$ and increased in an approximately linear fashion 0 as the velocity of stretch was increased up tow $400^{\circ} / \mathrm{sec}$. The maximum EMG was recorded ato the position of greatest muscle length - that is, in the position of extension of the knee in the case of the hamstrings and the fully flexed $\vec{F}$ position in the case of quadriceps muscle.

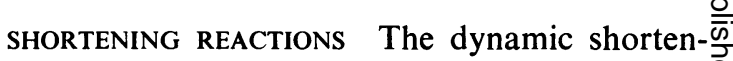
ing reaction was present in the triceps in all $\mathbb{\Phi}^{\mathbb{}}$ patients but was absent from the biceps muscle. In six of seven patients studied, the amplitude of $\overrightarrow{0}$ the shortening reaction of the triceps muscle was greater than the dynamic stretch reflex at anc angular velocity of $200 \% \mathrm{sec}$. The dynamic shortening reaction was velocity-dependent? (Fig. 5).

A dynamic shortening reaction occurred in seven of the 10 patients in the hamstrings muscles and in three of the 10 patients in the을 quadriceps.

EFFECTS OF ACTIVATION During the process reinforcement, the static stretch reflex of bice

quadaiceps

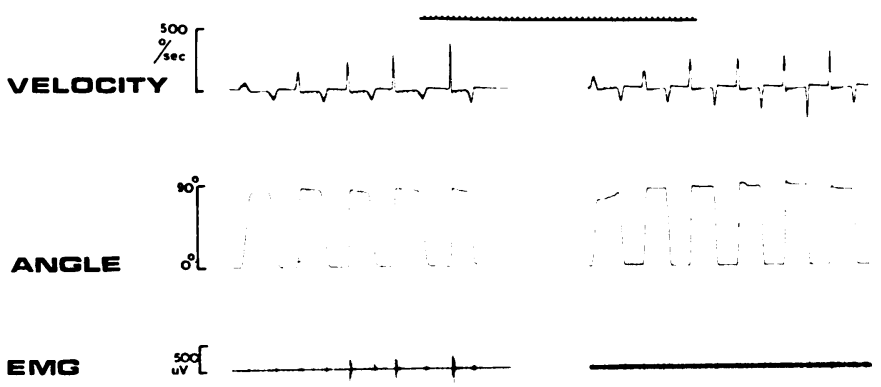

INT EMG ${ }^{\operatorname{cog}}[\ldots$
FIG. 6. The dynamic tonic stretch reflex of the quadriceps muscle before and during oral phenoxybenzamine therapy. EMG is absent at low velocities of stretch and then augments with increasing velocities of stretch in the control period. During phenoxybenzamine therapy the stretch reflex is absent. 


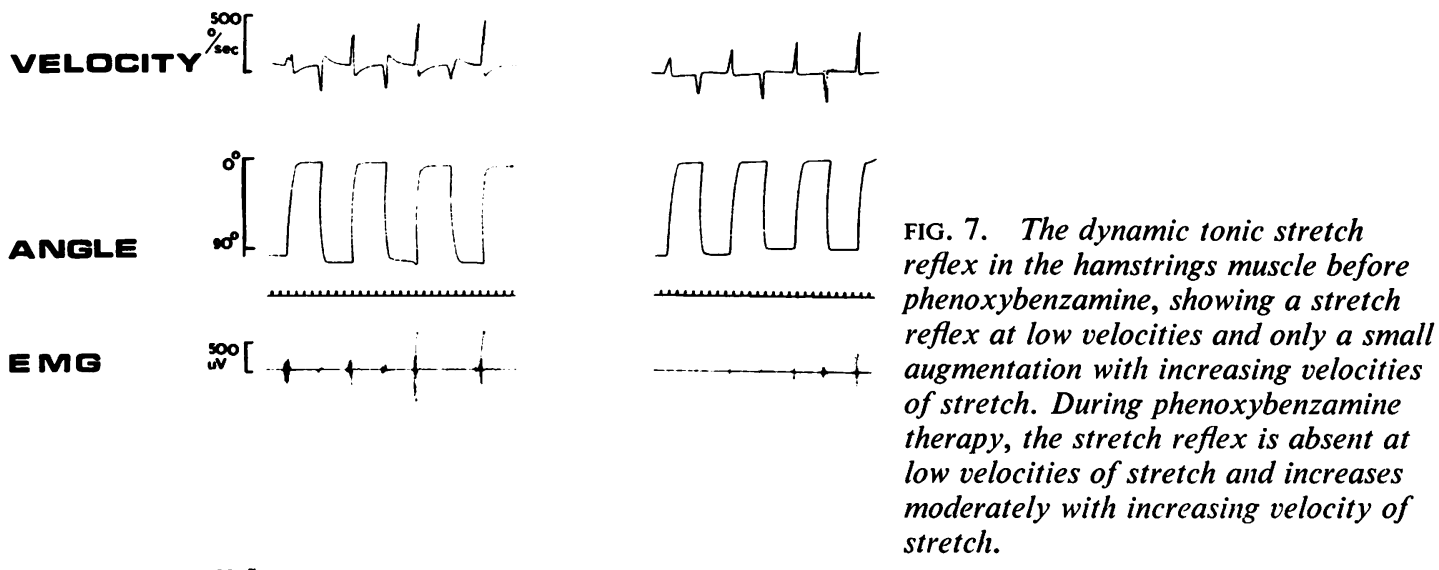

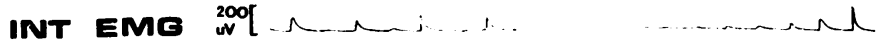

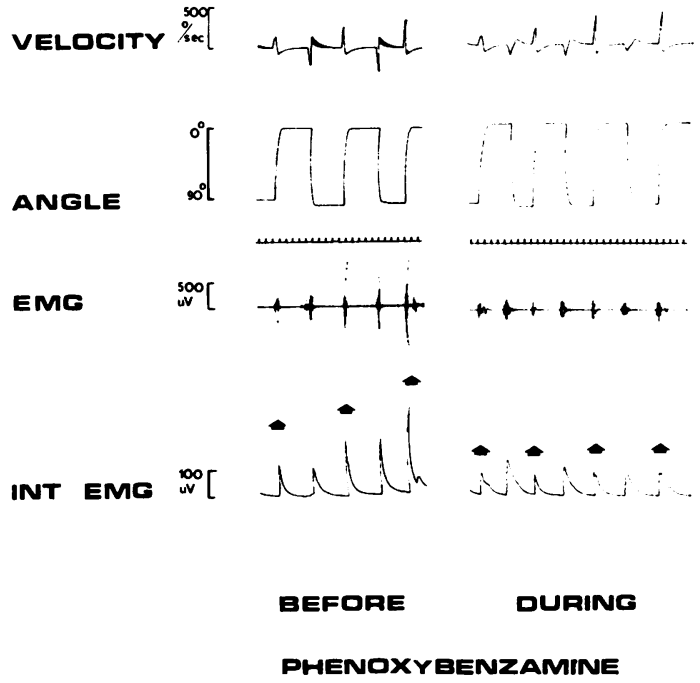

FIG. 8. Decrease in the dynamic sensitivity of the hamstrings stretch reflex (indicated with arrows) during phenoxybenzamine therapy while the amplitude of the stretch reflex is reduced. Also note a dynamic shortening reaction (without arrows). Movement artefact was excluded by monitoring each response on an oscilloscope. and triceps muscles increased more than the dynamic stretch reflexes. Both the static and dynamic stretch reflexes of biceps and triceps muscles remained maximal in the flexed position of the elbow joint.

The dynamic shortening reaction of the triceps was augmented in seven of eight patients during activation (Fig. 4), particularly at low velocities of shortening. Activation did not induce a dynamic shortening reaction in the biceps muscle.

Activation did not enhance the dynamic stretch reflexes or shortening reactions of hamstrings or quadriceps muscles, but a static stretch reflex appeared in the tracings of the hamstrings of three patients and of the quadriceps muscles of four patients.

EFFECT OF ISCHAEMIA OF ELBOW JOINT AND FOREARM Joint position sense in the elbow and wrist was blocked in one patient by an inflated cuff above the elbow joint for $35 \mathrm{~min}$. The discomfort caused by the inflated cuff had an activating effect but the phase relationships of the stretch reflex and shortening reactions remained unchanged.

EFFECT OF PHENOXYBEnZAMINE Clinical exam- 
ination of muscle power disclosed no alteration during periods of treatment with placebo or phenoxybenzamine. There was no improvement in the athetoid movements evoked by voluntary activity. In no patient was a drop in blood pressure of $20 \mathrm{~mm}$ of mercury or more recorded on standing from the supine position, but one patient admitted to postural faintness on direct questioning. One patient complained of fullness in the nose and five were mildly lethargic, mainly in the mornings, while taking phenoxybenzamine. No drowsiness or depression was experienced.

Stretch reflexes Phenoxybenzamine was effective in reducing the static stretch reflex in the biceps and triceps muscles but had little effect on the dynamic stretch reflex in these muscles, whereas the dynamic stretch reflex was reduced in both quadriceps and hamstrings muscles.

During the period of treatment with phenoxybenzamine the dynamic stretch reflex of the triceps muscle remained virtually unchanged. Phenoxybenzamine abolished the static stretch reflex of the triceps muscle in the one patient in whom it had been present during the placebo period.

The dynamic stretch reflex of the hamstrings muscle was maximal in the most stretched position during both placebo and phenoxybenzamine treatment periods.

Shortening reactions In six of seven patients the dynamic shortening reaction of the triceps muscle was reduced by phenoxybenzamine (Fig. 5). A static shortening reaction occurred in the triceps muscle of one patient only and was abolished during the phenoxybenzamine period.

In six patients, 10 recordings of the static response of the biceps muscle at $90^{\circ}$ of flexion of the elbow joint were averaged. In five of the six patients the static response was reduced to $50 \%$ or less during the phenoxybenzamine period as compared with the placebo period.

Involuntary movements Each patient was found to have his own characteristic pattern of involuntary movement, but no changes in power spectrum could be attributed to the effects of phenoxybenzamine.
Activation The effect of activation on the stati shortening reaction of the biceps muscle during placebo and phenoxybenzamine periods was compared in six patients. Phenoxybenzamineo was effective in reducing the increment in the resting activity of the biceps muscle in five of the six patients when the level of reinforcement, a judged by the EMG of the voluntary contraction: of the contralateral limb, was comparable fo $\overrightarrow{\vec{p}}$ both periods.

\section{DISCUSSION}

An electromyographic study of reflex musculañ activity in 10 patients with the athetosis $\vec{\circ}$ spasticity syndrome has disclosed a patteri which differs considerably from that of hemio paretic spasticity and of Parkinson's diseases (Table 2).

The biceps dynamic stretch reflex was in hibited by increasing muscle length, unlike that of spasticity and Parkinson's disease. This demonstrated by measuring the amplitude of stretch reflex at different muscle lengths durB sinusoidal stretching and also by observing phase relationships during sinusoidal stretchigge The phase lead of the biceps stretch reflex found to be greater than $90^{\circ}$, supporting conclusion from systematic sinusoidal stretch at different joint angles that the net effect

TABLE 2

DIFFERING PATTERNS OF REFLEX MUSCULAR ACTIVITY

\begin{tabular}{|c|c|c|c|}
\hline & $\begin{array}{c}\text { Hemiparetic } \\
\text { spasticity }\end{array}$ & $\begin{array}{c}\text { Parkinson's } \\
\text { disease }\end{array}$ & Athetosis \\
\hline \multicolumn{4}{|l|}{ Biceps brachii } \\
\hline Stretch reflex & $\begin{array}{l}\text { Mainly } \\
\text { dynamic }\end{array}$ & $\begin{array}{l}\text { Dynamic and } \\
\text { static }\end{array}$ & Mainly static \\
\hline Effect of length & Facilitatory & Facilitatory & Inhibitory \\
\hline \multicolumn{4}{|l|}{ Triceps brachii } \\
\hline Stretch reflex & $\begin{array}{l}\text { Mainly } \\
\text { dynamic }\end{array}$ & $\begin{array}{l}\text { Dynamic and } \\
\text { static }\end{array}$ & Dynamic \\
\hline Effect of length & Facilitatory & Facilitatory & Facilitatory \\
\hline \multicolumn{4}{|l|}{ Hamstrings } \\
\hline Stretch reflex & $\begin{array}{l}\text { Mainly } \\
\text { dynamic }\end{array}$ & $\begin{array}{l}\text { Dynamic and } \\
\text { static }\end{array}$ & Dynamic \\
\hline Effect of length & Facilitatory & Facilitatory & Facilitatory \\
\hline \multicolumn{4}{|l|}{ Quadriceps } \\
\hline Stretch reflex & $\begin{array}{l}\text { Mainly } \\
\text { dynamic }\end{array}$ & $\begin{array}{l}\text { Dynamic and } \\
\text { static }\end{array}$ & Dynamic \\
\hline Effect of length & Inhibitory & Facilitatory & Facilitatory \\
\hline
\end{tabular}


increasing muscle length is inhibition of the stretch reflex. The possibility of joint receptors contributing to this inhibition was eliminated by the lack of change in the phase relationships during ischaemic block of joint afferent nerve fibres.

The fact that continuous activity can be recorded in the biceps muscle at rest in the shortened position suggests that the flexed dystonic posture is produced by a state of alpha motoneurone hyperactivity. Muscle stretch introduces a number of reflex segmental mechanisms which may be facilitatory or inhibitory to the alpha motoneurone. Present concepts of these mechanisms are derived from the spinal cat, which may not be applicable to normal or athetotic man.

The triceps stretch reflex was velocitydependent and facilitated by length in the same way as it is in Parkinson's disease and spasticity. The stretch reflex of hamstrings and quadriceps muscles was velocity-dependent, like that of spasticity, but differed from spasticity in that the quadriceps stretch reflex was facilitated by increasing muscle length, instead of being inhibited to produce the clasp-knife phenomenon.

The length facilitation and velocity dependence of the stretch reflex of triceps, hamstrings, and quadriceps muscles is in agreement with predominant group Ia afferent fibre activity from these muscles and one does not have to postulate any other factors to explain the pattern of the reflex response to passive stretch.

There are good grounds for considering that the length-dependent inhibition responsible for the clasp-knife phenomenon of spasticity is caused by the release from bulbospinal control of flexor reflex afferent pathways, particularly those from group II afferent fibres (Burke et al., 1970). This phenomenon is found in the quadriceps muscle of spastic patients but not in the flexors or extensors of the upper limbs (Ashby and Burke, 1971). In the present series of athetotic patients, length-dependent inhibition was found in the biceps brachii but not in the quadriceps muscle. Since the biceps brachii is an anti-gravity muscle in man, comparable in this respect with the quadriceps muscle in the lower limb, it appears as though group II afferent fibre effects are released in the upper limb of athetotic man.
It seems unlikely that the involuntary movements of athetosis are directly related to the rigido-spasticity, because patients with severe athetotic movements commonly had only mild rigido-spasticity and the involuntary movements were not reduced when the rigido-spasticity diminished during treatment with phenoxybenzamine. Narabayashi and co-workers (1965) have classified athetosis on the basis of electromyographic assessment into rigid and rigidospastic types. This study reveals that spasticity (in the sense of high dynamic sensitivity) is usually found in the triceps, quadriceps, and hamstrings muscles, whereas rigidity (low dynamic sensitivity) is found in the biceps muscle.

Foerster (1921) described a tonic spasm occurring when a muscle is passively shortened; this appears to be the same phenomenon as the dynamic shortening reaction observed in this study, which is particularly prominent in the triceps muscle. In the upper limb, the shortening reactions are equally as prominent as the stretch reflexes and, indeed, the dynamic shortening reaction of the triceps muscle is usually greater in amplitude than the dynamic stretch reflex. The dynamic shortening reaction was most prominent in the triceps muscle of athetotic patients and a similar pattern occurs in activated normal people, patients with Parkinson's disease (Andrews, Burke, and Lance, 1972), and dystonia musculorum deformans (Yanagisawa and Goto, 1971). This similarity suggests a common mechanism in these four conditions.

Activation in normal people has been studied by the use of the Jendrassik manoeuvre (Gassel and Diamontopoulos, 1964), by the application of cold, and the injection of adrenaline (Landau, Struppler, and Mehls, 1966) and by hand clenching (Mark, 1963). Reinforcement probably involves activation of both alpha and gamma efferent fibres (Gassel and Diamantopoulos, 1964) but, in the lower limb at least, the fusimotor system is probably more important (Buller and Dornhorst, 1957). Activation in normal man produces predominantly static stretch reflexes (Landau, Struppler, and Mehls, 1966) and personal observations have shown that the static electromyographic activity in the biceps muscle is maximal in the flexed position. The similarities between the reflex characteristics 
of activated normal man and the athetotic patient at rest suggests that in athetosis central inhibition of the normal reinforcement mechanisms is impaired. The method of activation used in this study was effective in facilitating the static but not the dynamic component of the tonic stretch reflex and this response is consistent with increased static fusimotor activity.

Phenoxybenzamine, an alpha-adrenergic blocker, was found to be effective in reducing static stretch reflexes, the dynamic shortening reaction in the triceps and the static shortening response in the biceps muscle, which are the reflexes augmented by activation. Moreover, the effect of activation during the phenoxybenzamine period was diminished as judged by the static activity of the biceps muscle. Noradrenaline, iontophoretically applied to neurones in the reticular formation, excites some neurones and inhibits others. Chlorpromazine selectively inhibits the neurones excited by iontophorectically applied noradrenaline and Bradley, Wolstencraft, Hösli, and Avanzino (1966) postulate that the adrenolytic effects of chlorpromazine may be important in its mode of action as a tranquillizer. It is possible that phenoxybenzamine and chlorpromazine have similar adrenolytic effects in the reticular formation and that the responses observed above during activation are partly dependent on noradrenaline.

Considering the actions of the descending noradrenergic system explained in the introduction of this paper, phenoxybenzamine therapy would be expected to decrease the stretch reflex of extensors but to have a variable effect in flexor muscles, which was born out by this study. While this work was in progress, a reference was noted to the intravenous use of thymoxamine, an alpha adrenergic blocking agent, in the relief of spasticity (Lancet, 1970). The result of the present double-blind controlled trial of phenoxybenzamine in athetosis adds support to the claim that alpha-adrenergic blocking agents may be useful in spasticity. The descending noradrenergic system has only a small projection to the forelimb in the cat (Andén et al., 1966). If this be the same in man, it could account for the differences in the response to phenoxybenzamine of the dynamic stretch reflexes in upper and lower limbs. This study suggests that activation or arousal plays only a small part in the involuntary movements of athetosis.

The authors wish to thank Mr. and Mrs. Edwin Street, the National Health and Medical Research Council of Australia and the Spastic Centre of $\stackrel{?}{工}$ N.S.W. for their financial support of this project. Associate Professor James W. Lance has provided invaluable assistance in the preparation of the manu- $\vec{F}$ script. The placebo capsules were supplied by Smith, Kline, and French and the random allocation of $\frac{}{2}$ phenoxybenzamine and placebo was arranged by Miss A. M. Mackie, chief pharmacist of The Prince Henry Hospital. We also wish to thank Sister P. $\cong$ Sears, sister-in-charge, Centre Industries. The के illustrations were prepared by the Department of $\overrightarrow{0}$ Medical Illustration, University of New South Wales.

\section{REFERENCES}

Andén, N.-E., Jukes, M. G. M., and Lundberg, A. (1966). ఝु The effect of DOPA on the spinal cord. 2. A pharmaco logical analysis. Acta Physiologica Scandinavica, 67, 3875 397.

Andén, N.-E., Jukes, M. G. M., Lundberg, A., and Vyklick呑 음 L. (1966). The effect of DOPA on the spinal cord. 3. DQ $\rightarrow$ polarization evoked in the central terminals of ipsilater $T$ Ia afferents by volleys in the flexor reflex afferents. $A C T$ G Physiologica Scandinavica, 68, 322-336.

Andrews, C. J., Burke, D., and Lance, J. W. (1972). T度 response to muscle stretch and shortening in parkinsoniam rigidity. Brain, 95, 795-812.

Ashby, P., and Burke, D. (1971). Stretch reflexes in the upper limb of spastic man. Journal of Neurology, Neurosurgery, and Psychiatry, 34, 765-771.

Baker, R. G., and Anderson, E. G. (1970). The effects of L-3,4-dihydroxyphenylalanine on spinal reflex activity. Journal of Pharmacology and Experimental Therapy, 173, 212-223.

Bergman, P. S., Nathanson, M., and Bender, M. B. (1953). Effect of intravenous barbiturates on abnormal involuntary movements. Neurology (Minneap.), 3, 503-512.

Bergmans, J., and Grillner, S. (1968). Changes in dynamic sensitivity of primary endings of muscle spindle afferents induced by DOPA. Acta Physiologica Scandinavica, 74, 629-636.

Blackman, R. B., and Tukey, J. W. (1959). The Measurement of Power Spectra. Dover: New York.

Bradley, P. B., and Wolstencroft, J. H. (1965). Actions of drugs on single neurones in the brain-stem. British Medical Bulletin, 21, 15-18.

Bradley, P. B., Wolstencroft, J. H., Hösli, L., and Avanzino, G. L. (1966). Neuronal basis for the central action of chlorpromazine. Nature, 212, 1425-1427.

Buller, A. J., and Dornhorst, A. C. (1957). The reinforcement of tendon-reflexes. Lancet, 2, 1260-1262.

Burke, D., Andrews, C. J., and Gillies, J. D. (1971). The reflex response to sinusoidal stretching in spastic man. Brain, 94, 455-470.

Burke, D., Gillies, J. D., and Lance, J. W. (1970). The N quadriceps stretch reflex in human spasticity. Journal of $\mathrm{N}$ Neurology, Neurosurgery, and Psychiatry, 33, 216-223. 
Carlsson, A., Falck, B., Fuxe, K., and Hillarp, N.-Å. (1964). Cellular localization of monoamines in the spinal cord. Acta Physiologica Scandinavica, 60, 112-119.

Corrodi, H., Fuxe, K., Lidbrink, P., and Olson, L. (1971). Minor tranquillizers, stress and central catecholamine neurons. Brain Research, 29, 1-16.

Dahlström, A., and Fuxe, K. (1965). Evidence for the existence of monoamine neurons in the central nervous system. 2. Experimentally induced changes in the intraneuronal amine levels of bulbospinal neuron systems. Acta Physiologica Scandinavica, 64, Suppl. 247.

Foerster, O. (1921). Zur Analyse und Pathophysiologie der striären Bewegungsstörungen. Zeitschrift für die gesamte Neurologie und Psychiatrie, 73, 1-169.

Gassel, M. M., and Diamantopoulos, E. (1964). The Jendrassik maneuver. 1. The pattern of reinforcement of monosynaptic reflexes in normal subjects and patients with spasticity or rigidity. Neurology (Minneap.), 14, 555-560.

Grillner, S. (1969). The influence of DOPA on the static and dynamic fusimotor activity to the triceps surae of the spinal cat. Acta Physiologica Scandinavica, 77, 490-509.

Herz, E. (1931). Die amyostatischen Unruheerscheinungen.
Klinischkinematographische Analyse ihrer Kennzeichen und Begleiterscheinungen. Journal für Psychologie und Neurologie, 43, 3-182.

Hoefer, P. F. A., and Putnam, T. J. (1940). Action potentials of muscles in athetosis and Sydenham's chorea. Archives of Neurology, 44, 517-531.

Jenkins, G. M., and Watts, D. G. (1968). Spectral Analysis and its Applications. Holden-Day: San Francisco.

Lancet. Drug treatment of spasticity. Lancet, 2, 918-919.

Landau, W. M., Struppler, A., and Mehls, O. (1966). A comparative electromyographic study of the reactions to passive movement in Parkinsonism and in normal subjects. Neurology (Minneap.), 16, 34-48.

Mark, R. F. (1963). Tonic stretch reflexes in the calf muscles of normal human subjects. Nature, 199, 50-52.

Narabayashi, H., Nagahata, M., Nagao, T., and Shimazu, H. (1965). A new classification of cerebral palsy based upon neurophysiologic considerations. Confinia Neurologica, 25, 378-392.

Yanagisawa, N., and Goto, A. (1971). Dystonia musculorum deformans. Analysis with electromyography. Journal of Neurological Science, 13, 39-65. 